\title{
Der wissenschaftliche Film ${ }^{1}$ )
}

Von Dr.-Ing. G. Wour

Direktor der Abteilung Hochschule und Forschung

des Instituts für Film und Bild, Göttingen

Meine Herren Präsidenten, Magnifizenzen, Herr Generaldirektor, verehrte Gäste!

Es ist mir eine besondere Ehre und Freude, Sie in so großer Zahl im Namen des Instituts für Film und Bild bei uns willkommen zu heißen. Mir ist es zunächst ein Bedürfnis, für die freundlichen Worte und Wünsche zu danken, die der Herr Vertreter des Niedersächsischen Kultusministeriums überbrachte, sowie für das Grußwort, das uns die Technische Hochschule Hannover im Auftrage ihres Rektors übermittelte. Es sind nun 6 Jahre her, seitdem sich in Deutschland zum letztenmal ein größeres Gremium zur Besprechung von Fragen des wissenschaftlichen Films versammelt hatte; und während in Laufe der letzten 4 Jahre in allen Kulturstaaten des Auslandes staatliche Institute für den wissenschaftlichen Film wieder aufgebaut oder neu gegründet wurden, waren bei uns in Deutschland erhebliche Schwierigkeiten zu überwinden, um die Arbeit in kleinstem Rahmen fortführen zu können. Daß es dann möglich wurde, die Existenz zu sichern, haben wir der verständnisvollen Einsicht der westdeutschen Kultusminister zu danken, die sich davon überzeugten, daß die zentrale Zusammenfassung des wissenschaftlichen Filmes in Form eines staatlichen Institutes ja nicht das Ziel hat, zusätzlich Geld auszugeben, sondern Kosten einzusparen. Es ist uns ein Bedürfnis, für diese verständnisvolle Entscheidung auch heute zu danken.

Nachdem die finanzielle Grundlage der Arbeit gesichert erschien, mußte ein Weg gefunden werden, die Abteilung Hochschule und Forschung aus der dörflichen Umgebung zu befreien, in die sie 4 Wochen vor der Kapitulation als verlagerter Teil der ehemaligen Reichsanstalt für Film und Bild gelangt war. Hier war es die MaxPlanck-Gesellschaft, durch deren besonderes Entgegenkommen wir geeignete Räume erhielten, und für das wir ihrem Präsidenten und ihrem Generaldirektor zu besonderem Dank verpflichtet sind.

1) Referat, gehalten anläßlich der Jahrestagung der Hochschulfilmreferenten am 27.10.1949 in Göttingen. 
Und wenn wir heute nun zum ersten Male wieder in größerem Rahmen in Erscheinung treten können, haben wir auch Ihnen, meine Damen und Herren, zu danken, die Sie als Vertreter der Militärregierungen, sowie der verschiedenen Ministerien hier anwesend sind, und Ihnen, die aus den verschiedenen Teilen Westdeutschlands, aber auch aus Berlin, zu uns gekommen sind, um in Referaten $\mathrm{zu}$ uns zu sprechen. Insbesondere sind wir aber auch glücklich, Sie meine Herren Filmreferenten als Vertreter der 30 westdeutschen Hochschulen bei uns zu haben, um heute nachmittag im internen Kreise für den Wiederaufbau der wissenschaftlichen Filmarbeit eine geeignete Grundlage zu erarbeiten.

Die Entstehung des wissenschaftlichen Films geht bis in die ersten Anfänge der Kinematographie zurück - ja, die Grundlagen der Kinematographie sind zum großen Teil durch Wissenschaftler geschaffen worden, die das Ziel verfolgten, ein Gerät zu konstruieren, um Bewegungsvorgänge bildmäßig aufzulösen und sie dem Auge wieder darzubieten.

1874 konstruiert der französische Astronom JANSSEN den photographischen Revolver, mit dem er in 48 Einzelaufnahmen den Venusdurchgang vor der Sonne erfaßt.

1877 macht der Amerikaner MUYBRIDGE Bewegungsstudien an laufenden Tieren mit Hilfe von 24 Kameras.

1882 konstruiert der französische Physiologe MAREY seine photographische Flinte für Reihenaufnahmen vom Vogelflug.

1888 berichtet er der Pariser Akademie der Wissenschaften über erfolgreiche Reihenaufnahmen auf einem ruckweise bewegten Negativband.

1890 macht MaREy Mikroaufnahmen.

1891 arbeitet KoHLRAUSCH, Hannover, an einem aus 24 Kameras zusammengesetzten Gerät mit einem besonderen Parallaxenausgleich.

Im gleichen Jahr wird von Braune und Fischer, Leipzig, ein von MAREY angegebenes Verfahren der Reihenphotographie durch Mehrfachbelichtung zu der klassisch gewordenen Arbeit über den Gang des Menschen benutzt.

1894 werden von MAREY erstmalig Hochfrequenzaufnahmen bis zu 120 Bildern in der Sekunde aufgenommen. Noch ist die Projektion nicht gefunden, und die Aufnahmen werden mit dem Lebensrad projiziert. 
Uberall sind es zum großen Teil Wissenschaftler, die führend bei der Ent wicklung der Kinematographie beteiligt sind. - Dann scheint eine Zeit zu kommen, wo dieses Interesse zurückgeht. Auf den Rummelplätzen und Jahrmärkten laufen die ersten Projektoren; die Kinematographie wird zum Massenunterhaltungsmittel. Der Vorstadtkintopp entsteht. Man scheint sich auf Seiten der Wissenschaftler etwas zu genieren, mit diesem Apparat zu tun zu haben. Trotzdem, - neben dem immer mächtiger werdenden Strom des beginnenden Spielfilms fließt ein kleines, langsam wachsendes Bächlein für die Wissenschaft. - Noch vor dem ersten Weltkriege entsteht in Frankreich das Institut Marey, nach dem Kriege in Deutschland das Medizinisch-Kinematographische Institut in Berlin, dann die Reichsanstalt für Film und Bild, deren Arbeiten auf wissenschaftlichem Gebiet durch die hiesige Abteilung Hochschule und Forschung des Instituts für Film und Bild fortgeführt werden. In allen bedeutenden Staaten entstehen staatliche Institute für den wissenschaftlichen Film. Im Jahre 1947 wird in Paris eine International Scientific Film Association gegründet.

Bereits bei den ersten meist physiologischen Untersuchungen, die mit Hilfe der Kinematographie durchgeführt werden, ist das Prinzipielle gut zu erkennen. Die Methode erlaubt als Forschungsfilm Bewegungsvorgänge zu analysieren und damit grundlegende Erkenntnisse zu schaffen; die so gefundenen Erkenntnisse werden mit den Aufnahmen als Beleg auf Kongressen und vor den wissenschaftlichen Akademien vorgeführt und schließlich auch im Hochschulunterricht verwendet. Damit war die erste Entwicklungsstufe des Hochschulunterrichtsfilmes erreicht. - Danach ist der manchmal etwas unklar gewordene Begriff ,,wissenschaftlicher Film" auch klar umrissen. In Analogie zum wissenschaftlichen Buch und zur wissenschaftlichen Zeitschrift ist der wissenschaftliche Film ein Film für der Wissenschaft. Ein Kulturfilm über ein wissenschaftliches Thema ist in diesem Sinne kein wissenschaftlicher Film, sondern ein populär-wissenschaftlicher Kulturfilm.

An die Arbeiten jener längst verstorbenen Pioniere der wissenschaftlichen Kinematographie knüpft die Arbeit unseres Institutes bewußt an und verfolgt als Hauptaufgabe den Forschungs- und den Hochschulunterrichtsfilm.

Wenden wir uns zunächst einmal dem Forschungsfilm zu. Die Kinematographie benutzt dabei das Grundprinzip der Photographie mit deren Möglichkeiten der optischen Abbildung, der 
Sichtbarmachung und der optischen Fixierung eines augenblicklichen Zustandes.

Das (optische) Abbild gibt objektiv einen Tatbestand wieder. Das Studium dieses Abbildes kann neue forschungsmäßige Erkenntnisse erbringen. Darüber hinaus wird, da die abbildende Optik bestimmten Gesetzen folgt, eine weitgehende meßmäßige Auswertung ermöglicht (Photogrammetrie).

Die Möglichkeit der Sichtbarmachung, also einer Mehrleistung gegenüber dem menschlichen Auge, ergibt sich durch die Verwendung von Emulsionen mit anderen Empfindlichkeitsbereichen oder durch Kopplung der Photographie mit anderen optischen Methoden und Anordnungen.

Die optische Fixierung hat den Vorteil, daß der in dem Abbild fixierte Tatbestand immer wieder und unverändert für die Zwecke des Studiums, der Auswertung und des Vergleiches zur Verfügung steht. Damit stellt das photographische Abbild des Tatbestandes gleichzeitig ein Dokument dar und kann als Beleg Verwendung finden.

Dieselben Gesichtspunkte gelten zunächst auch theoretisch für die Kinematographie, aber nun nicht mehr für die Erfassung eines augenblicklichen Zustandes, sondern eines Bewegungsvorganges Es tritt durch die zusätzliche Erfassung des Faktors Zeit ein neues entscheidendes Moment hinzu.

Um einen Bewegungstorgang durch subjektive optische Beobachtung analysieren zu können. ist es erforderlich. daß der zeitliche Ablauf dieses Vorganges in einem Geschwindigkeitsbereich liegt, der durch das Auge erfaßt werden kann. Wohl die meisten Vorgänge der Natur und eine große Anzahl aller technischen Vorgänge liegen jedoch außerhalb dieses Bereiches, d.h. sie sind in ihrer Ablaufgeschwindigkeit zur Erfassung durch das menschliche Auge zu schnell oder zu langsam. Lm den Vorgang nun durch die optische Beobachtung untersuchen zu können, ist es demnach erforderlich, die Ablaufgeschwindigkeit in den für die Beobachtung geeigneten Bereich zu verlegen. Praktisch die einzige Methode hierfür bietet die Kinematographie mit Zeitdehner und Zeitraffer.

Die Veränderbarkeit des Zeitmaßstabes, die der Wissenschaft damit zur Verfügung steht, bietet eine ebensolche Erweiterung der Erkenntnismöglichkeiten in zeitlicher Hinsicht, wie sie das Mikro skop und das Fernrohr in räumlicher Hinsicht erbrachten. 
$\mathrm{Zu}$ diesen grundsätzlichen Möglichkeiten treten nun andere hinzu. Durch Benutzung verschiedener Bereiche der elektro-magnetischen Wellenskala können unter Verwendung geeigneter Emulsionen nicht nur das sichtbare Licht, sondern Infrarot-, Ultraviolett-, Röntgen- oder Elektronenstrahlen zur Abbildung benutzt werden oder Aufnahmen mit polarisiertem, monochromatischem oder Fluoreszenz-Licht durchgeführt werden. Man kann fernerhin die Kinematographie koppeln mit anderen Methodon, z. B. dem Mikroskop, dem Teleskop, der Schlierenmethode zur Erfassung an sich unsichtbarer Medien und deren Bewegungen. dem Stroboskop und dem Spektroskop. und wir können. wie bekannt, Ton- und Farbaufnahmen durchführen. S.hließlich können, die so gewonnenen Aufnahmen in verschiedener Richtung ausgewertet werden. Es ist dabei die subjektir beobacintende Bewegungserfassung von der objektiv messenden Bewegungsanalyse, der Meßkinematographie zu unterscheiden. mit deren Hilfe man Zeitmessungen, Weg- und Entfernungsmessungen. Zeit-Weg-Diagramme und Geschwindigkeitsmessungen durchführen kann, und die sich in speziellen Fällen $z u$ einer Kinematogrammetrie entwickeln läßt.

Die sinnvolle Kopplung aller dieser einzelnen Verfahren untereinander gibt dann erst einen vollständigen Eindruck von der Fülle der Möglichkeiten, die die kinematographische Forschungsmethode für die Wissenschaft bietet. Selbstverständlich gibt sie keine forschungsmäßige Patentlösung. Sie ist in den meisten Fällen ein Glied in der Kette der anzuwendenden Methoden, deren Ergebnisse ausgewertet werden müssen, um das Problem zu lösen. Aber es ist ebenso Tatsache, daß unzählige Forschungsvorhaben Jahre und Jahrzehnte nicht vorankamen, eben weil dieses Glied in der ganzen Forschungskette fehlte.

Was sind das nun für Forschungsfilmaufgaben, die im Augenblick im Vordergrund stehen? Nun, es handelt sich dabei z. B. um die Erfassung des Verhaltens lebender menschlicher Krebszellen bei der Teilung oder bei der Beeinflussung durch chemische oder physikalische Methoden. - Mehrere Wissenschaftszweige sind auf der Auswertung biologischer Mikropräparate aufgebaut. Wie sich jedoch das Dauerpräparat zur ursprünglich lebenden Zelle verhält, ist noch weitgehend ungeklärt. Auch hier wird der Forschungsfilm zu neuen Erkenntnissen beitragen. In der Bodenkunde wird er wesentliche Ergebnisse bringen, bei der klimatisch bedingten Veränderung der Bodenstrukturen, der Krümelbildung usw. - Selbstverständlich sind das nur einige Beispiele für viele. 
Bei denjenigen Aufgaben, die die Hochschulen und Forschungsinstitute nicht selbst durchführen können, sehen wir es als unsere Aufgabe an. die geeigneten Methoden zu finden und mit einem minimalen Aufwand den gewünschten Erfolg zu erzielen.

Eigene Forschungs- und Entwicklungsaufgaben liegen nun in der sinnvollen Weiterentwicklung von Geräten und Verfahren. Es ist dabei leicht einzusehen, daß jede Verbesserung in dieser Richtung neue forschungsmäßige Möglichkeiten erschließt. Eine Weiterentwicklung der Hochfrequenzaufnahmeverfahren für Vorderlicht über jene kritische Grenze hinaus, die gerade noch von mechanischen Zeitdehnern aufgenommen werden können, wird für viele Fragen der Mechanik und Technik wichtig sein. Ein Röntgenblitzzeitdehner wird es z. B. ermöglichen, manche Vorgänge im Innern laufender Maschinen zu untersuchen. - Die Auswertverfahren müssen verbessert und vereinfacht werden, um den wissenschaftlichen Instituten eigene Auswertarbeiten zu erleichtern. Die Erhöhung der Meßgenauigkeit, ebenso wie die Definierung der auftretenden Fehler, wird zu einem vermehrten Einsatz der Meßkinematographie führen.

Im ganzen muß angestrebt werden, die im allgemeinen für einen ganz anderen $Z$ weck, nämlich für den Spielfilm geschaffenen Geräte und Verfahren für die Wissenschaft geeignet zu machen. Aus den kinematographischen Apparaturen müssen wissenschaftliche Instrumente, aus den kinematographischen Verfahren wissenschaftliche Verfahren werden. -

Wie wir sahen, entstand zusammen mit dem Forschungsfilm der Hochschulunterrichtsfilm, und während in Schaubuden und im Panoptikum Filmstreifen zum Amüsement vorgeführt wurden und von einem künstlerischen Spielfilm noch lange keine Rede sein konnte, führte der Franzose Dorex 1898 auf dem Ärztekongreß in Edinburgh die ersten medizinischen Filme vor. 1899 machte Pfeffer, damals Direktor des Botanischen Instituts der Universität Leipzig, seine Zeitrafferaufnahmen vom Wachstum der Pflanze und Messter stellte zur gleichen Zeit in Berlin die ersten deutschen medizinischen Filme her. Biologie und Medizin blieben dann für Jahrzehnte die großen Hauptgebiete des Hochschulfilms, bis später die Technik als dritter großer Anwendungsbereich in Erscheinung trat. Der Verlauf einer Operation ist im Kolleg deutlicher im Film als in Wirklichkeit nahezubringen. Die Verhaltensweise eines Tieres in freier Natur oder unter dem Mikroskop einem größeren Kreise - also im Unterricht - zu verdeutlichen, 
ist durch den Film in besonders guter Weise möglich. Die Anschaulichkeit des bewegten Bildes macht ihn für den Unterricht wertvoll. Ist es bei dem Forschungsfilm die Erfassung und Auswertung des zu analysierenden Vorganges, so ist es bei dem Hochschulunterrichtsfilm die pädagogische Gestaltung, die im Mittelpunkt der Aufgabe steht. Wie bei einer sprachlichen Formulierung eines wissenschaftlichen Referates oder einer Publikation sorgfältig abgewogen werden muß, so ist das Gleiche bei der pädagogischen Gestaltung eines Unterrichtsfilmes notwendig. Einstellung, Kameraführung, Blenden, Schnitt sind solche Mittel der Gestaltung. Ein besonders sorgfältig zusammengestelltes Manuskript ist Voraussetzung. Zahlreiche praktische Fragen sind zu beantworten: Wie muß ein wissenschaftlicher Film gestaltet sein, um ein Maximum an pädagogischer Wirkung zu erzielen, wie lang darf er sein, in welchem Umfang müssen $Z$ wischentitel erscheinen, wie geschieht am besten die unterrichtliche Auswertung, wo müssen Ergänzungen durch Trickteile berücksichtigt werden.

Wir wissen, daß der Film, auch der wissenschaîtliche Film, zunächst psychische Bezirke, dann erst den Intellekt anspricht. ,Zunächst überredet der Film, er überzeugt erst bei bestimmter Gestaltung“", hat ZIEROLD früher einmal formuliert. Dieser Umweg über die Psyche scheint sich aber auch bei Filmen, die der wissenschaftlichen Wissensvermittlung dienen, eher vorteilhaft als nachteilig auszuwirken. Solche und andere psychologische Fragestellungen werden in umfangreichen Forschungsarbeiten behandelt werden müssen, um hier Grundlagen zu schaffen. In Frankreich ist es das Institut de Filmologie an der Sorbonne, das hier bereits Pionierarbeit geleistet hat, jedoch den wissenschaftlichen Film unseres Wissens noch nicht berücksichtigte.

- Hochschulunterrichtsfilme werden durch das Institut nur in engster Zusammenarbeit mit den Hochschullehrern hergestellt und auch erst dann, wenn von einem Gremium von Hochschullehrern der betreffenden Fachrichtung ein solcher Film für unbedingt erforderlich gehalten wird. Die wissenschaftliche Leitung und Verantwortung liegt genau wie bei dem Forschungsfilm bei dem Hochschulinstitut, die filmwissenschaftliche Bearbeitung und Gestaltung und die technische Durchführung bei dem Filminstitut.

Die Hochschule soll ja nun nicht nur eine Stelle der Forschung und der Wissensvermittlung sein, sondern sie soll nach abendländischem Ideal den ganzen Menschen formen, ihn innerlich bilden. 
Wir haben häufig bei der ersten Vorführung von Forschungsaufnahmen erlebt, wie kühle Wissenschaftler nicht nur in laute Begeisterung ausbrachen, sondern in staunender Verwunderung blieben. Sollte man diese Möglichkeit eines inneren Erlebnisses, denn das ist es ja wohl, nicht in menschenbildender Richtung einsetzen können? Freilich gibt der Film nur ein Abbild der Natur wieder, aber er macht objektiv sichtbar, was sonst dem Auge verschlossen bliebe, und das nicht einem Einzelnen, sondern einer Gemeinschaft. - Auch dem wissenschaftlichen Film kommt ein Erlebnismoment zu. Es liegt nicht im stimmungsmäßigen, sondern im forschungsmäßigen Miterleben, - einem Faktor von besonderer erzieherischer Bedeutung. Bild - Sinnbild - und Bildung haben ja nicht ohne inneren Grund denselben Stamm. - Solche Filme könnten durchaus in der Richtung mitwirken, die von der Denkschrift zur Hochschulreform als anzustrebendes Ziel für das studium generale gefordert wird. Sie würden dem Goetheschen Schauen entsprechen müssen, das ja nicht nur naturwissenschaftliche Erfassung, sondern auch künstlerische Betrachtung und inneres Erleben bedeutet.

Nun, wir haben noch keinen solchen Film, aber es sind Ansätze dazu im Ausland und auch bei uns vorhanden, und es wird wertvoll sein. diese Aufgabe im Auge zu behalten. -

Nachdem wir kurz die Möglichkeiten des Forschungsfilmes und des Hochschulunterrichtsfilmes erläutert haben. bleibt die Frage, was getan werden kann, um diese Möglichkeiten bei den Hochschulen auf breiter Basis zu fördern.

Zunächst dürfen wir darauf hinweisen, da 3 das Institut die Selbstherstellung von Filmaufnahmen durch die Hochschulinstitute in jeder Weise fördern wird. Auf dem Forschungsgebiet, speziell der Zeitdehnung, wird diese Selbstherstellung auch in Zukunft beschränkt sein, denn die erforderlichen (ieräte sind zu teuer. Ein Hochfrequenzzeitdehner kostet etwa $20000,-$ DM, ist in Deutschland nur noch in wenigen Exemplaren vorhanden und wird bei uns auch zur Zeit nicht mehr hergestellt. Zur Bedienung der Geräte gehören Spezialisten. Der Film, der in 2 Sekunden durch den Apparat läuft, kostet 50,- DM. Mehrfache Versuche sind also kostspielig. Für einzelne Aufgaben wird man sicher schon Schmalaufnahmegeräte benutzen können, die mit dem nötigen Zubehör relativ viel billiger sind. Bei schwierigeren Aufnahmen wird es jedoch zweckmäßig sein, sich der Beratung oder des Aufnahme- 
dienstes des Institutes zu bedienen, dessen wissenschaftlich geschultes Aufnahmepersonal über die notwendigen Erfahrungen verfügt.

Bei dem Hochschulunterrichtsfilm liegen die Verhältnisse ähnlich. Auch hier wird die Initiative der Institute, Aufnahmen selbst durchzuführen, z. B. bei den medizinischen Fallsammlungen, bei psychologischen und tierpsychologischen Aufnahmen usw. gefördert werden. - Der gestaltete Standard-Hochschulunterrichtsfilm wird im Regelfall eine Angelegenheit des Zentralinstitutes bleiben.

Einen Hinweis glauben wir nicht vorenthalten zu dürfen. Im Laufe der letzten 20 Jahre sind außerhalb der Institutsarbeit für die Herstellung wissenschaftlicher Filme durch Hochschul- und Forschungsinstitute, Kliniken, Expeditionen und bei anderen $\mathrm{Ge}$ legenheiten hohe Kosten entstanden. Von allen dafür geschaffenen Vorhaben ist mit geringen Ausnahmen nichts mehr verblieben. Entweder waren es ungenügende Erfahrungen bei der Aufnahme, Mangel an Gestaltung, Nichtübertragung von Rechten, unsachgemäße Aufbewahrung, das Fehlen von Dublikaten, überhaupt das Fehlen einer Archivierung, die dann dazu führten, da $B$ dieses Material der Wissenschaft verlorenging. - All diese Schwierigkeiten können weitgehend vermieden werden, wenn es zu einer guten $\mathrm{Zu}$ sammenarbeit zwischen dem Institut und den Hochschulfilmreferenten kommt. Wir versprechen Ihnen, von uns aus alles zu tun, was dieser Gemeinschaftsarbeit dient.

Lassen Sie mich bitte noch kurz auf die Verhältnisse im Ausland eingehen. In Europa bestehen Institute für den wissenschaftlichen Film in Frankreich, Holland, Belgien, Polen und Italien. Schweden baut zur Zeit ein solches auf. In Großbritannien besteht eine British Scientific Film Association, in den Vereinigten Staaten mehrere Laboratorien für den wissenschaftlichen Film, ein großes Zentralinstitut wird zur Zeit aufgebaut. In Rußland bestehen, wie mir gelegentlich des vorjährigen ISFA-Kongresses der russische Delegierte persönlich sagte, wegen der Größe des Landes fünf wissenschaftliche Filminstitute. Das Interesse des Auslandes an dieser Arbeit ist außerordenlich rege. Bei dem Anfang Oktober dieses Jahres in Brüssel stattgefundenen diesjährigen Kongreß der ISFA waren 32 Staaten durch Delegierte vertreten. Der wichtigste Beschluß dieses Kongresses war die Schaffung eines Forschungskomitees, in dem neben Frankreich, England, Holland, Belgien, Österreich und Italien auch Deutschland Mitglied ist. Eine Tagung 
dieses internationalen Ausschusses für die Forschungs-Kinematographie wird im nächsten Jahr hier in Göttingen in unserem Institut stattfinden. -

Im Ganzen sind wir der Meinung, daß unsere Arbeit ein Anfang ist, wie wir hoffen ein guter. Gerade in der heutigen Zeit, mit ihrer Notwendigkeit, die Forschung zu fördern, werden dem Forschungsfilm wichtige Aufgaben zukommen. Der Film als Methode der Wissensvermittlung und der Erziehung wird an den Hochschulen nicht mehr wegzudenken sein. Beide bieten uns Möglichkeiten, die wir in vollem Umfange bisher nicht übersehen können, deren Förderung für uns eine große und verantwortungsvolle, aber auch beglückende Aufgabe darstellt. 
Druck: Hubert \& Co., Göttingen 